\title{
NK cells for PD-1/PD-L1 blockade immunotherapy: pinning down the NK cell
}

\author{
Cordelia Dunai' and William J. Murphy ${ }^{1,2}$ \\ 'Department of Dermatology and 'Department of Internal Medicine, School of Medicine, UCD, Sacramento, California, USA.
}

\begin{abstract}
In spite of a very robust body of literature and definitive data demonstrating the importance of the programmed cell death receptor-1 (PD-1) pathway in T cells and their function, the data on NK cell PD-1 expression have been highly variable and, particularly in the case of mouse NK cells, scarce. In this issue of the JCI, Hsu et al. present data demonstrating PD-1 expression on mouse NK cells only within tumors and show that PD-1 blockade elicits an antitumor NK cell-mediated response. This study indicates that, given the complexity of both the biology and study of NK cells, further work is needed to more clearly determine the role of the PD-1/PD-1 ligand (PD-L1) on NK cells.
\end{abstract}

\section{Complex nature of NK cells}

NK cells are an innate lymphoid cell population that have been extremely challenging to both characterize and therapeutically exploit since their first functional description in the 1960s as radioresistant cells capable of mediating bone marrow allograft rejection in lethally irradiated mice (1). NK studies have been, and still are, hampered by inadequate markers allowing clear delineation from $\mathrm{T}$ cells and other cell types coupled with significant differences between mouse and human NK cells with regard to phenotype (e.g., mice do not express CD56 commonly used to identify human NK cells), receptor expression (notably, the mouse Ly49 and human KIR MHC-binding regulatory receptors), and tissue distribution (human CD56 ${ }^{\text {bright }} \mathrm{NK}$ cells are present in lymph nodes, but NK cells are relatively absent in resting mice) (2). A huge hurdle for defining in vivo properties solely attributable to NK cells is reliance on NK cell-targeting markers also present on other cell types (notably T cells, NKT cells, and innate lymphoid cells), which significantly complicate conclusions regarding definitive roles.
While there are very strong evolutionary evidence and data associating NK cells and viral resistance (notably CMV), it is the well-described ability of NK cells to mediate non-MHC-restricted killing of tumor cells that makes them attractive for cancer therapy. However, while NK cells can play potentially significant roles in the resistance to some hematologic malignancies (3), preclinical evidence for and clinical trials with adoptive transfer for use against solid tumors have had rather disappointing results, in part due to a still incomplete understanding of the biology and complex regulation of these heterogeneous cell types $(4,5)$. This is likely multifactorial and related to their functional dependency on high cytokine exposure (particularly after extensive ex vivo culturing and expansion to increase numbers), inability to home to the tumor sites (NK cells primarily reside in hematologic tissues and liver), and putative shorter longevity (the actual life span of an NK cell is still unclear). This makes understanding and use of these cells much more complex than initially surmised, with subpopulations sometimes exhibiting antagonistic immunological functions $(5,6)$. Nonethe-

Related Article: p. 4654

less, given the recent dramatic successes of checkpoint blockade targeting the programmed cell death receptor-1 (PD-1) and its ligand (PD-L1) in cancer and the strong similarities between NK and T cells, there is considerable interest in targeting PD-1 to increase NK activities. However, NK cell expression of PD-1 has been difficult to understand. Only one-quarter of healthy human donors studied have a small percentage of $\mathrm{PD}-1^{+}$peripheral blood $\mathrm{NK}$ cells (interestingly, all positive donors were $\mathrm{CMV}^{+}$) (7). There is increasing evidence of $\mathrm{PD}-1$ expression on small subsets of NK cells in cancer patients (7-9), and in vitro induction of PD-1 on NK cells has been reported (10). This is in marked contrast to both mouse and human T cells, in which TCR crosslinking leads to a uniform and higher PD-1 expression. Unlike what occurs with T cells, NK cell function and numbers appear primarily controlled by the cytokine environment, which is further regulated by the need for transpresentation of IL-15 (11). NK cell anergy has been reported with continuous IL-15 exposure in vivo, and inhibitory molecules such as TIGIT have been shown to suppress NK cell function (Figure 1), but the extent to which inhibitory pathways affect overall responses is still unclear (Figure 1 and refs. 12-14). There are also extensive potent inhibitory receptors (Ly49/KIR, NKG2A) that can markedly affect NK cell function due to MHC and associated molecule binding (14). These observations raise the question of how and why PD-1 is involved in NK cell responses, although induction of PD-L1 expression on activated NK cells has also been shown (15).

\section{Involvement of PD-1 pathway and NK cells in cancer}

The PD-1/PD-L1 pathway may indeed play a functional role with NK cells, either directly or indirectly, but there may be differences in this regard between mouse and human. PD-1 expression on human NK cells after ex vivo expansion 
A

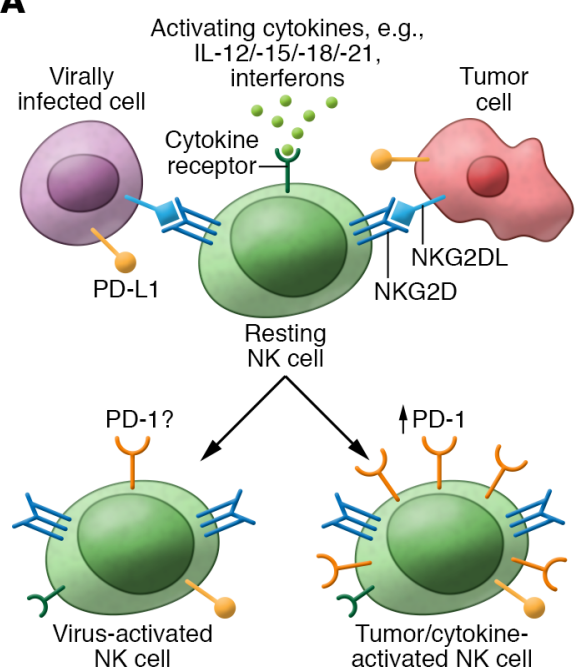

B

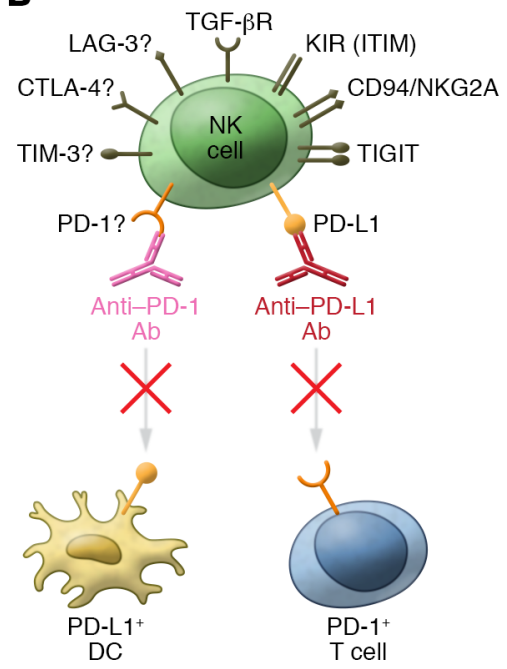

Figure 1. The role of NK cells in checkpoint blockade. (A) Inflammatory host disease environments affect PD-1 expression on NK cells. Hsu et al. show upregulation of PD-1 expression in the tumor microenvironment. The signals that determine PD-1 expression on NK cells are not completely understood. Checkpoint blockade could activate NK cells by preventing inhibition mediated by PD-L1+ target cells. (B) The activity of NK cells is determined by an array of activating and inhibitory signals, only a small selection of which are shown here. Antibody blockade of PD-1 would prevent cell-intrinsic inhibitory signaling. Antibody blockade of PD-L1 would also prevent cell-intrinsic signaling and, additionally, prevent NK cells from sending an inhibitory signal to PD-1+ $\mathrm{T}$ cells and/or PD-L1+ antigenpresenting cells (DCs).

has been reported, and two publications have shown that NK cell-stimulating treatments combined with PD-1 blockade augmented survival in mouse tumor models $(10,16,17)$. Importantly, there have not been extensive reports of PD-1 expression on mouse NK cells after mouse CMV infection despite robust activation. At present, given the paucity of definitive studies identifying PD-1 on NK cells, key questions still remain: What factors induce PD-1 expression on NK cells, and why only on a fraction of the population? What is the physiologic role of PD-1/PD-L1 on NK cell biology, given that both can be expressed? (18) Why are data showing expression of PD-1 on NK cells after viral infection in mice lacking? Is PD-1 expressed on NK cells inhibitory, and what functions/parameters are affected? Are there species differences between mouse and human NK cells with regard to PD-1? Finally, can PD-1/PD-L1 blockade directly or indirectly augment NK cell function?

The current study by Hsu et al. examines this issue by assessing the role of PD-1 on mouse NK cells in various tumor models (19). The authors demonstrate that NK subpopulations did appear to

express PD-1, but with a very important caveat - only when NK cells within tumors were examined. They further show that PD-L1-transduced tumor cells suppressed immune resistance and that removal of NK cells abrogated antitumor efficacy of PD-1 blockade. The data by Hsu et al. thus suggest that NK cells are potential responders to checkpoint blockade (19). Importantly, this effect on PD-1 induction, although heterogeneous, could be observed in multiple tumor models using two inbred strains of mice.

\section{Future directions}

Although these data have potentially significant implications for cancer immunotherapy, including in strategies to enhance the clinical benefit of NK cells, several critical factors must first be taken into consideration. First and foremost, the bulk of the in vivo tumor data used antibodies to globally deplete NK cells to verify that the effects observed could be specifically attributed to them. Therein lies a potential problem. The use of asialo GM1 (ASGM1) glycolipid as an NK-specific marker is extremely problematic, as ASGM1 is present on multiple cell types, including activated $\mathrm{T}$ cells and macrophages $(20,21)$
This key caveat was dramatically illustrated by a study over 30 years ago that reported that the effect of anti-ASGM1 in preventing graft-versus-host disease (GVHD) in mice was due to the depletion of NK cells (22). Subsequent studies by the same group found this was in fact due to activated T cells expressing ASGM1 (23). Even NK1.1, a marker commonly used to deplete NK cells that is more specific, is expressed on other cell types (2). To label an agent as NK specific in mice has always been problematic, particularly in the case in which $\mathrm{T}$ cells can be affected, and necessitates the need for $\mathrm{T}$ cell-deficient mice and/or adoptive transfer studies using purified NK cells. This is particularly important, as the data by Hsu et al. clearly show a significant contribution by $\mathrm{T}$ cells following antiPD-L1 treatment in some of the tumor models (19). Another consideration concerns the extensive use of subcutaneous tumor models and the actual extent of NK cell infiltration compared with that of $\mathrm{T}$ cells, which is compounded by the difficulty arising from phenotyping immune cells within a tumor. This is highlighted by the extreme heterogeneity of PD- $1^{+} \mathrm{NK}$ cells, which can range from $2 \%$ to $70 \%$ positive in some tumors within a single mouse cohort. Enzymatic digestion of the tumor with collagenases or the presence of proteases produced by granulocytes and other cells can have effects on downmodulating mouse CD 3 components $(24,25)$, which, if used as the sole distinguisher, could mask possible T cell PD-1 expression. Even with these caveats, taking these data with other recent studies, there are indications that the PD-1 pathway may indeed play a role in NK cell biology that could be exploited. As we understand more about NK cell biology, it is tempting to speculate that "memory-like" NK cells express PD-1, perhaps during chronic CMV infection, in parallel with long-lived $\mathrm{PD}-1^{+}$memory human $\mathrm{T}$ cells. In that regard, it is surprising that this induction has not been observed after systemic mouse NK cell activation, such as in viral infections (i.e., mouse CMV) or even in vitro following coculture with cytokine-transduced tumor feeder lines, but that may be contingent on time points assessed. It is important to note that checkpoint blockade also potentially affects PD-L1 on the activated NK cell and that this may be another possible mecha- 
nism for the increased antitumor effects. As we learn about both mouse and human NK cell biology, it will be important to incorporate the various immunoregulatory pathways, possibly including PD-1/PD-L1, with respect to NK cell function into clinical practice, as both direct and indirect effects may be observed.

\section{Acknowledgments}

We would like to thank Robert Canter, Dan Longo, and members of the Murphy lab for helpful discussion.

Address correspondence to: William J. Murphy, University of California, Davis Medical Center, 2921 Stockton Blvd., IRC Building, Room 1614, Sacramento, California 95817, USA. Phone: 916.703.9397; Email: wmjmurphy@ucdavis.edu.

1. Cudkowicz G, Stimpfling JH. Hybrid resistance to parental marrow grafts: association with the $\mathrm{K}$ region of H-2. Science. 1964;144(3624):1339-1340.

2. Zamora AE, Grossenbacher SK, Aguilar EG, Murphy WJ. Models to study NK cell biology and possible clinical application. Curr Protoc Immunol. 2015;110:14.37.1-14.37.14.

3. Ruggeri L, et al. Effectiveness of donor natural killer cell alloreactivity in mismatched hematopoietic transplants. Science. 2002;295(5562):2097-2100.

4. Grossenbacher SK, Aguilar EG, Murphy WJ. Leveraging natural killer cells for cancer immunotherapy. Immunotherapy. 2017;9(6):487-497.

5. Knorr DA, Bachanova V, Verneris MR, Miller JS. Clinical utility of natural killer cells in cancer therapy and transplantation. Semin Immunol. 2014;26(2):161-172.
6. Gill S, et al. Rapid development of exhaustion and down-regulation of eomesodermin limit the antitumor activity of adoptively transferred murine natural killer cells. Blood. 2012;119(24):5758-5768.

7. Pesce S, et al. Identification of a subset of human natural killer cells expressing high levels of programmed death 1: A phenotypic and functional characterization. J Allergy Clin Immunol. 2017;139(1):335-346.e3.

8. Liu Y, et al. Increased expression of programmed cell death protein 1 on NK cells inhibits NK-cellmediated anti-tumor function and indicates poor prognosis in digestive cancers. Oncogene. 2017;36(44):6143-6153.

9. Beldi-Ferchiou A, et al. PD-1 mediates functional exhaustion of activated NK cells in patients with Kaposi sarcoma. Oncotarget. 2016;7(45):72961-72977.

10. Lieberman NAP, et al. An uncoupling of canonical phenotypic markers and functional potency of ex vivo-expanded Natural Killer cells. Front Immunol. 2018;9:150.

11. Waldmann TA. The biology of interleukin-2 and interleukin-15: implications for cancer therapy and vaccine design. Nat Rev Immunol. 2006;6(8):595-601.

12. Stanietsky N, et al. The interaction of TIGIT with PVR and PVRL2 inhibits human NK cell cytotoxicity. Proc Natl Acad Sci U S A. 2009;106(42):17858-17863.

13. Felices M, et al. Continuous treatment with IL-15 exhausts human NK cells via a metabolic defect. JCI Insight. 2018;3(3):96219.

14. Beldi-Ferchiou A, Caillat-Zucman S. Control of NK cell activation by immune checkpoint molecules. Int J Mol Sci. 2017;18(10):E2129.

15. Iraolagoitia XL, et al. NK cells restrain spontaneous antitumor $\mathrm{CD}^{+} \mathrm{T}$ cell priming through PD-1/PD-L1 interactions with dendritic cells. JImmunol. 2016;197(3):953-961.

16. Guo Y, et al. PD1 blockade enhances cyto- toxicity of in vitro expanded natural killer cells towards myeloma cells. Oncotarget. 2016;7(30):48360-48374.

17. Bezman NA, et al. PD-1 blockade enhances elotuzumab efficacy in mouse tumor models. Blood Adv. 2017;1(12):753-765.

18. Zhao Y, Harrison DL, Song Y, Ji J, Huang J, Hui E. Antigen-presenting cell-intrinsic PD-1 neutralizes PD-L1 in cis to attenuate PD-1 signaling in T cells. Cell Rep. 2018;24(2):379-390.e6.

19. Hsu J, et al. Contribution of NK cells to immunotherapy mediated by PD-1/PD-L1 blockade. JClin Invest. 2018;128(10):4654-4668.

20. Nishikado H, Mukai K, Kawano Y, Minegishi Y, Karasuyama H. NK cell-depleting antiasialo GM1 antibody exhibits a lethal offtarget effect on basophils in vivo. J Immunol. 2011;186(10):5766-5771.

21. Wiltrout RH, et al. Reactivity of anti-asialo GM1 serum with tumoricidal and nontumoricidal mouse macrophages. J Leukoc Biol. 1985;37(5):597-614

22. Charley MR, Mikhael A, Bennett M, Gilliam JN, Sontheimer RD. Prevention of lethal, minordeterminate graft-host disease in mice by the in vivo administration of anti-asialo GM1. J Immu nol.1983;131(5):2101-2103.

23. Charley MR, Mikhael A, Hackett J, Kumar V, Bennett M. Mechanism of anti-asialo GM1 prevention of graft-vs-host disease: identification of allo-antigen activated T cells. J Invest Dermatol. 1988;91(3):202-206.

24. Franco JL, et al. Partial degradation of T-cell signal transduction molecules by contaminating granulocytes during protein extraction of splenic $\mathrm{T}$ cells from tumor-bearing mice. Cancer Res. 1995;55(17):3840-3846.

25. Mizoguchi H, O’Shea JJ, Longo DL, Loeffler CM, McVicar DW, Ochoa AC. Alterations in signal transduction molecules in T lymphocytes from tumor-bearing mice. Science. 1992;258(5089):1795-1798. 